\title{
In-situ Mechanical Characterization of Abdominal Muscle Using Stereo Imaging
}

\author{
Raquel Simón-Allué ${ }^{1}$, Antonio Agudo², J.M.M. Montiel ${ }^{2}$, Begoña Calvo ${ }^{1}$ \\ ${ }^{1}$ Grupo de Mecánica Aplicada y Bioingeniería (AMB) \\ ${ }^{2}$ Grupo de Robótica, Percepción y tiempo real (ROPERT) \\ Instituto de Investigación en Ingeniería de Aragón (I3A). \\ Universidad de Zaragoza, Mariano Esquillor s/n, 50018, Zaragoza, Spain. \\ Tel. +34-976762707, Fax +34-976762043, e-mail: rsimon@unizar.es
}

\begin{abstract}
We present a method to characterize in vivo the mechanical behavior of abdominal muscle, using inverse finite element analysis and image processing algorithms to estimate the properties. The aim of this technique is to help surgeon to choose the hernia mesh that better reproduces the mechanical behavior of the healthy muscle.
\end{abstract}

\section{Material and methods}

\section{Experimental data}

A total of six inflation tests were developed on New Zealand rabbits, half on healthy rabbits and other half on previous treated rabbits, with a defect inside of $7 \times 5 \mathrm{~cm}$. The inner pressure during the test varies from 0 to $8 \mathrm{mmHg}$.

All experiments were recorded using a stereo imaging system and techniques multi-view stereo [1] to estimate the 3D reconstruction of the rabbit surface. The accuracy obtaining with this method was $1 \mathrm{~mm}$. As the surface is textureless, we paint artificial markers to set up correspondences. Taking the $3 \mathrm{D}$ coordinates of the points at the null pressure state, the geometry of the whole cavity was reconstructed in order to obtain an initial validate model for a FEM simulation (see Figure 1).

\section{Numerical analysis}

Once the geometry is defined, it was necessary to formulate a constitutive law that defines the mechanical behavior of the tissue. A common methodology for soft tissues is to postulate the existence of a Strain Energy Function (SEF) decoupled in volumetric and isochoric responses, which in turn can be divided in isotropic and anisotropic part [2]. Based on previous biaxial tests and according to the literature [3], three material models have been proposed to simulate the muscle behavior: Neo-Hookean model, dependent on one parameter, and Demiray and Mooney Rivlin models, dependent on two.
To determine the value of the constants, inverse analysis method was used [4]. A total of 500 simulations for each model were performed with Abaqus varying the parameters combinations in a specific range. For each simulation displacements of the numerical analysis $u_{\text {num }}$ and experimental results $u_{\text {exp }}$ were compared. Mean Square Error (MSE) (1) was defined as an objective function to evaluate the success of the numerical data.

$$
\begin{aligned}
& M S E=\sqrt{\frac{1}{N P} \cdot \frac{1}{n^{\circ} p t o s} \cdot\left[\sum_{i=1}^{N P} w_{i} \cdot \sum_{k=1}^{n^{\circ} p t o s}\left(e_{k}\right)^{2}\right]} \\
& e_{k}=\sqrt{\left(u_{\text {num }}-u_{\text {exp }}\right)_{x}^{2}+\left(u_{\text {num }}-u_{\text {exp }}\right)_{y}^{2}+\left(u_{\text {num }}-u_{\text {exp }}\right)_{z}^{2}}
\end{aligned}
$$

where NP means the pressure levels here analysed, $w_{i}$ the weight given to each level and $n^{o}$ ptos the number of points studied on the abdomen surface.

The whole process is described in the following diagram.

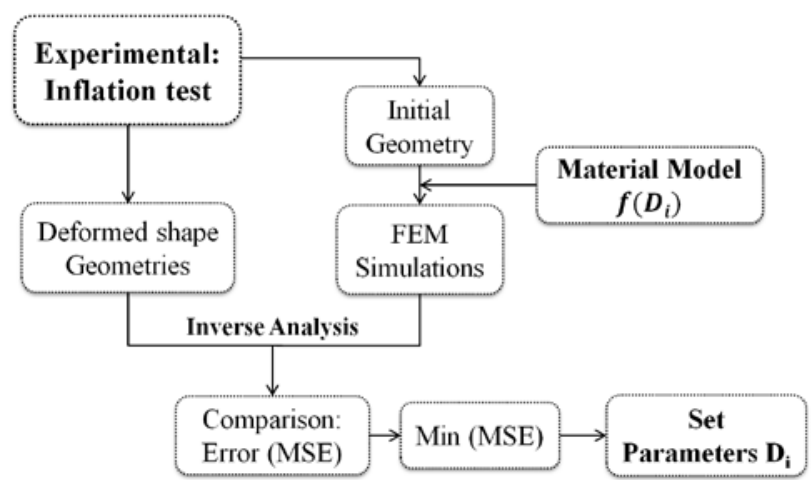

\section{Results and discussion}

A response surface of the MSE was plotted for each model. Results showed that all models present a zone in the response surface that minimizes the error made by the simulation. That zone corresponds to the parameters combination that better characterize the material behavior. The form 
of the minimum depends on the material model chosen.

While Demiray's model presented a specific point that minimizes the surface, Mooney-Rivlin's model showed a valley of combinations where all parameters sets minimize the MSE (see Figure 2). In the case of Neo-Hookean model, dependent on only one parameter, the solution was a line instead of a surface and presented a definitive minimum.

The deformed shape of those combinations which minimize the solution, coincides and reproduces the deformed configuration of the experimental test.

\section{Conclusions}

The procedure works successfully as far as it is able to find the specific parameters that minimize the error surface, and therefore, better reproduce the muscle behavior. However, some aspects of the FEM reconstruction must be improved, such as the boundary conditions or load application, in order to fully guarantee the total reproduction of the experimental test.

New material models and a more complex abdominal model must be evaluated before considering obtained paramenters as a solution.

\section{Acknowledgments}

This project has been possible thanks to the Spanish Ministry of Economy and Competitiveness, which supports this researching through the grant BES2012-053422 and the project DPI2011-27939-C0201.

\section{REFERENCIAS}

[1]. HARTLEY, R. and ZISSERMAN, A. Multiple View Geometry in Computer Vision. 2nd edition, Cambridge: Cambridge University Press, 2004.

[2]. HOLZAPFEL, G.A. Nonlinear Solid Mechanics. New York: Wiley, 2000

[3]. HERNANDEZ, B., PEÑA, E., PASCUAL, G., RODRÍGUEZ, M., CALVO, B., DOBLARÉ, M. and BELLÓN, J.M. Mechanical and histological characterization of the abdominal muscle. Journal of the Mechanical Behavior of Biomedical Materials. 2011, 4(3), 392-404.

[4]. KAUER, M., VUSKOVIC, V., DUAL, J., SZEKELY, G. and BAJKA, M. Inverse finite element characterization of soft tissues. Medical Image Analysis. 2002, 6(3), 275-287.

\section{Figures}

Figure 1: Estimating abdominal cavity geometry. (a): Arrangement of the stereo system. (b) Scene acquired. (c): Shape at rest reconstruction. (d): Initial FE mesh.

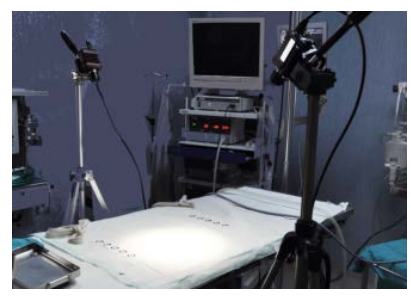

(a)

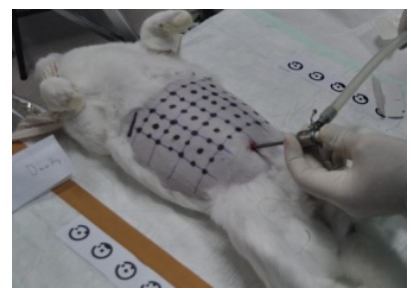

(b)

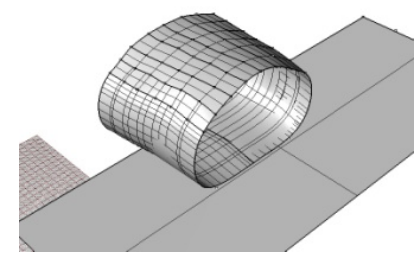

(c)

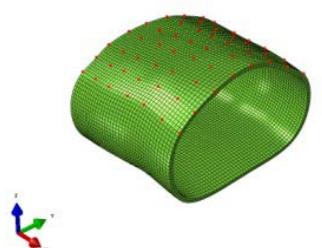

(d)

Figure 2: Results for one material model (Mooney-Rivlin) and comparison between models. (a) Response surface for Mooney-Rivlin model. (b) FEM simulation for Mooney-Rivlin, from 0 to $8 \mathrm{mmHg}$ of inner pressure. (c) Comparison of curves $\sigma-\lambda$ for the proposed models.

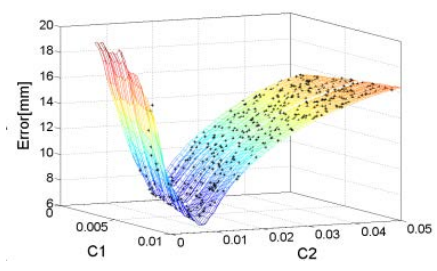

(a)

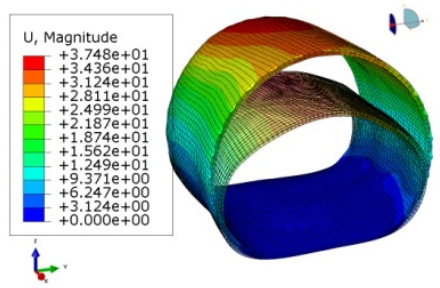

(b)

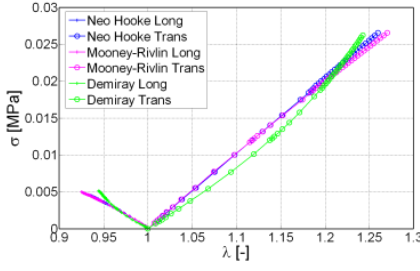

(c) 